https://doi.org/10.48009/2_iis_2007_97-102

\title{
DECISION MAKING TOOLS IN CELLULAR TELECOMMUNICATION NETWORK DESIGN: GIS AND NLP
}

\author{
Joon-Yeoul Oh, Texas A\&M University-Kingsville, joon-yeoul.oh@tamuk.edu \\ Jaehyung Yu, Texas A\&M University-Kingsville, kfjy000@tamuk.edu \\ Richard A. Aukerman, Texas A\&M University-Kingsville, richard.aukerman@tamuk.edu
}

\begin{abstract}
Using the correct tools to make a decision is very important to the decision makers. In this paper two supporting tools, Geographic Information Systems and Nonlinear Programming, are introduced to select the optimal location of a Mobile Telephone Switching Office in the cellular telecommunication network design. The cellular telecommunication network system often encounters capacity inadequacy issues due to an increase in the number of subscribers and the related data traffic. Installing a Mobile Telephone Switching Office (MTSO) is a common method used to increase the network capacity. Selecting the installation location of MTSO is critical due to its direct relationship to the installation cost and call routings. Candidate areas are selected using a Geographic Information Systems technique. A non-linear programming model is developed to solve the MTSO's optimal location problem of selecting a spot among the candidate sites, and two test cases are implemented and analyzed. Based on the analytical results of the test cases, this paper suggests a method of selecting MTSO locations along with the effectiveness of the method.
\end{abstract}

Keywords: Decision-making, Cellular Telecommunications, Network Design, Optimization, Non-linear Programming, Geographic Information Systems

\section{INTRODUCTION}

Most cellular phone users have had unpleasant experiences, such as disconnections or unstable and weak connections during conversations. In extreme rural areas, there may be limited or no reception, which can jeopardize customers' safety, especially during emergencies. This is due to the inadequate capacities of the cell site and the Mobile Telephone Switching Office (MTSO) designated to handle call volumes or call traffic in a cellular telecommunication.
According to the October 2005 issue of TWICE, there were 194.6 million cellular phone subscribers or $65 \%$ of the United States population. When compared to about $47 \%$ in June 2004 , the number has increased dramatically and is expected to continue increasing in the future [8]. If the existing communication network has inadequate capacity to comply with customer demands, a network expansion effort must be initiated.

To increase capacity, MTSOs may be deployed in the cellular network. If the existing MTSO's capacity is exceeded by the expanded network traffic or installing additional MTSOs is more economical, the installation location of the new MTSO must be determined. When determining the location, it is critical to consider connections between cell sites and MTSOs due to its direct impact on costs and call traffic routing. In this paper, two decision-making tools, Geographic Information Systems (GIS) and Nonlinear Programming (NLP), are used to determine the optimal location of the MTSO with consideration of common real world constraints such as costs and resources.

\section{OVERVIEW OF THE CELLULAR NETWORK COMMUNICATIONS}

When a cellular phone is turned on, it automatically connects to the tower with the strongest signal connection. The tower covers a certain area called a cell, so a cell site is composed of a cell and tower in the cell $[11,13]$. The tower (cell site) is connected to the handset (cellular phone) without a wired line to deliver the call. However, there is a wire connection between the cell site and a MTSO.

The MTSO is connected to other MTSOs, and one of the MTSOs (called home MTSO) is connected to the Public Switched Telephone Network (PSTN), which also allows your call to be delivered to the regular landline phones in your home or office $[6,10]$. The MTSOs (except the MTSO connected to PSTN) are often called hubs to distinguish them from the home MTSO. 


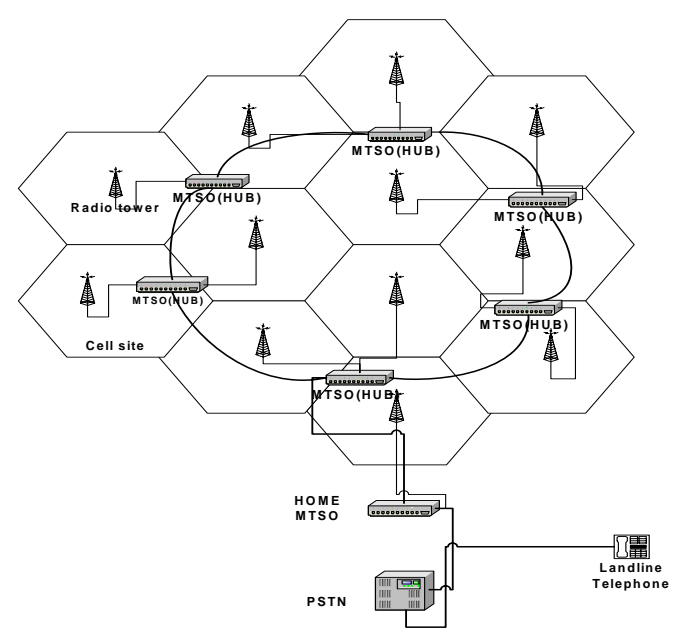

Figure 1: Rrief cellılar communication

Figure 1 shows a brief cellular network system containing essential components and their connections. Multiple cell sites are connected to one MTSO, and the MTSO is connected to other MTSOs. Usually, the connection of MTSOs is a ring topology allowing the call traffic to flow in another direction in case of a wire disconnection [4]. The capacity of the MTSO should meet or exceed the total capacity of cell sites connected to the MTSO. If the capacity of the MTSO is insufficient, especially during peak time, phone calls cannot be placed.

\section{CANDIDATE SITES SELECTION USING GEOGRAPHIC INFORMATION SYSTEMS}

The best MTSO location is determined based on two major processes; selecting candidate areas and finding the best location using optimization. Before executing the optimal location searching process, candidate areas, which are available for potential MTSO locations, should be identified based on the environmental regulations and economic efficiency. Geographic Information Systems (GIS) has been one of the most popular methods for various site selection studies due to its powerful functionalities in spatial data management [2,3]. It allows more efficient and effective spatial analysis for real world units since any spatial data dealt in a GIS environment are georeferenced to a map projected unit. In other words, the output candidate areas from GIS analysis will have very accurate geographic measurements such as perimeter and distance.

In general, candidate areas for MTSO must satisfy the following constraints:

1) Should avoid residential area and restricted areas such as military bases and wild life protection areas,

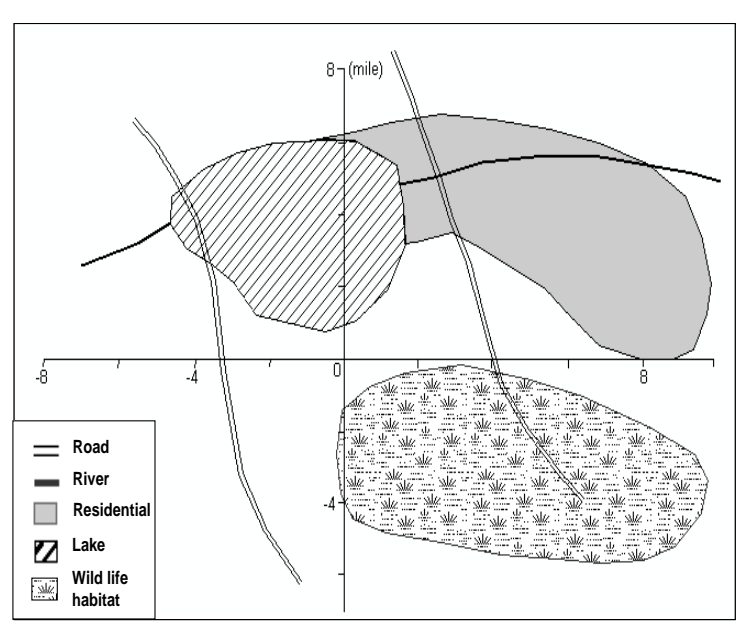

Figure 2: Base GIS layers

2) Should be easy to access from existing roads,

3) Should not be located in a high slope area, and

4) Should avoid proximity to bodies of water because of flooding.

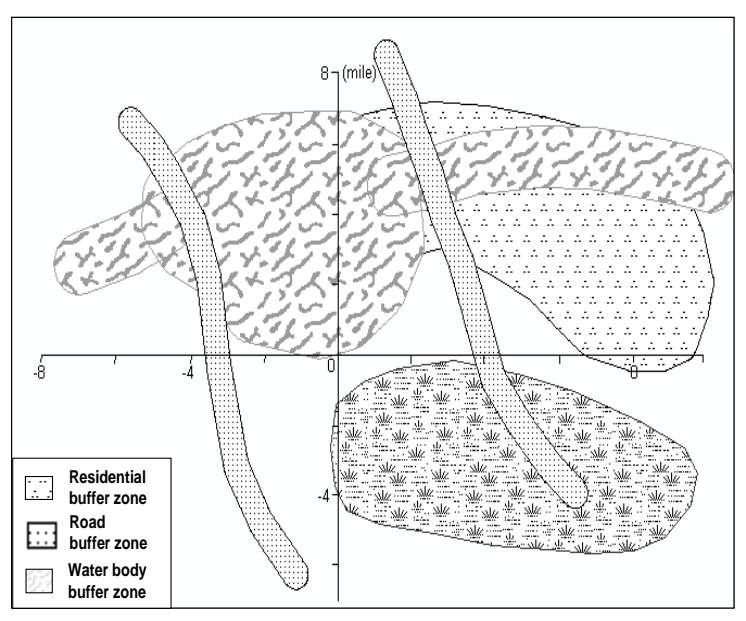

Figure 3: Buffer zones

To identify the best candidate sites satisfying the above constraints, first of all, each theme of GIS layers is added to the GIS environment (Figure 2), and the constraints are added up one by one using vector based buffering and overlay analysis [5]. In a simple example using ArcGIS ${ }^{\circledR}$ version 9.1, Figure 3 shows a 0.5 mile road buffer zone, 1 mile water body buffer zone, and 0.5 mile residential buffer zone which are created and mapped into the base GIS layers simulating constraints 2) and 4). The overlay analysis uses Boolean logic map operations by intersecting the "should be" constraints or erasing the "should not be" constraints, and provides an accurate map of candidate sites satisfying all the constraints 


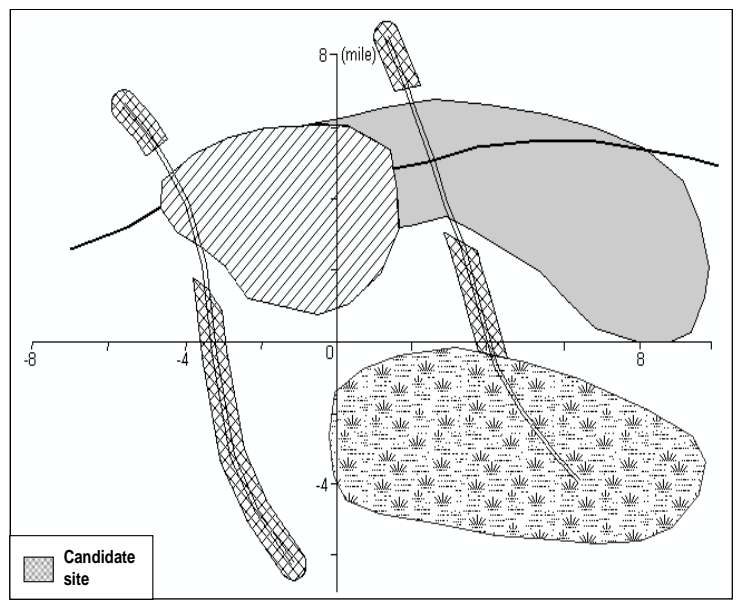

Figure 4: Selected candidate areas

(Figure 4). This result will be the input for selecting the best fitting location using the optimization technique.

\section{PROBLEM AND FORMULA FOR THE OPTIMAL LOCATION OF MTSO}

There are three major requirements in determining the best location for the MTSO:

1) Achievement of optimal connections between the MTSO and cell sites to minimize the connection costs,

2) Consideration of city expansion plans to avoid residential and restricted areas used in searching the candidate area, and

3) Accessibility of MTSO locations to effectively handle maintenance issues.

The location of MTSO should satisfy the requirements that can be formulated as a non-linear programming model. Let the coordinates of a point be $\left(x_{1}, y_{1}\right)$ and the coordinates of another point be $\left(x_{2}\right.$, $y_{2}$ ). Then, the distance between two points is $\sqrt{\left(x_{1}-x_{2}\right)^{2}+\left(y_{1}-y_{2}\right)^{2}}$ [12]. If a cellular network system requires a new MTSO, the objective is to find optimal connections between the MTSO and cell sites, and connections between the MTSO and other MTSOs. Based on the distance formula, the objective function can be formulated as a sum of the minimized distances since the distance is closely related to the installation cost and call traffic routing.

Let the coordinates of a MTSO be ( $m x, m y)$, the number of cell sites to connect to a MTSO be $j$ and the coordinates of cell sites be $\left(c x_{i}, c y_{i}\right)$, where $i=1$,
$2, \ldots, j$. The objective function is shown in the following.

$$
\text { Min } \begin{gathered}
\sqrt{\left(m x-c x_{1}\right)^{2}+\left(m y-c y_{1}\right)^{2}}+ \\
\sqrt{\left(m x-c x_{2}\right)^{2}+\left(m y-c y_{2}\right)^{2}}+ \\
\vdots \\
\sqrt{\left(m x-c x_{i}\right)^{2}+\left(m y-c y_{i}\right)^{2}} .
\end{gathered}
$$

Since the values, differences between two points, in the square roots are always positive, the objective function is differentiable and continuous at any point. Thus the objective function is convex. Since the set of solutions of the objective function is a nonempty convex set, a minimized solution is a global optimal solution [1]. In other words, the function is solvable, and a unique optimal solution exists.

The location of the MTSO must avoid specific areas because of the regulations, costs and installation difficulties. These restrictions are considered as constraints in the mathematical model. Let the number of avoiding areas be $l$ and the location of avoiding areas be $\left(a x_{k}, a y_{k}\right)$, where $k=1,2, \ldots, l$ and the required distance from an avoiding area be $d_{k}$. The value of a distance is a radius from the center of a restricted area to the longest edge. For this case, the constraints are formulated in the following manner.

$$
\begin{aligned}
& \text { Subject to } \sqrt{\left(m x^{2}-a x_{1}^{2}\right)+\left(m y^{2}-a y_{1}^{2}\right)} \geq d_{1} \\
& \sqrt{\left(m x^{2}-a x_{2}^{2}\right)+\left(m y^{2}-a y_{2}^{2}\right)} \geq d_{2} \\
& \sqrt{\left(m x^{2}-a x_{k}^{2}\right)+\left(m y^{2}-a y_{k}^{2}\right)} \geq d_{k}
\end{aligned}
$$

Because the square root of a square value is a convex function, the feasible solution set generated from the constraints, which are all convex functions, is a convex set. The objective values deteriorate if a point moves further in any direction from an original optimal solution [1, 7].

Using the Excel Solver ${ }^{\circledR}$, two different cases are tested: 1) without the candidate sites selection technique and 2) with the candidate sites selection technique. Five cell sites and three restricted areas are defined. Tables 1 and 2 show the numerical data used for the tests. Table 1 shows the coordinates of the locations of the MTSOs and/or cell sites, and Table 2 shows the coordinates of the center for the avoiding areas' locations in miles. The avoiding distances are the longest distance from the center to the edge of the avoiding area. 
Table 1: Locations of existing MTSOs and/or Cell Sites

\begin{tabular}{|l|l|l|}
\hline $\begin{array}{l}\text { MTSOs and/or Cell } \\
\text { Sites }\end{array}$ & $x$-axis & $y$-axis \\
\hline 1 & 3 & 6 \\
\hline 2 & 2 & -2 \\
\hline 3 & -7 & 7 \\
\hline 4 & -5 & -3 \\
\hline 5 & 8 & 3 \\
\hline
\end{tabular}

Table 2: Locations of avoiding areas

\begin{tabular}{|l|l|l|l|}
\hline Avoiding Area & $x$-axis & $y$-axis & $\begin{array}{l}\text { Avoiding } \\
\text { Distance }\end{array}$ \\
\hline 1 & -2 & 3 & 5 \\
\hline 2 & 5 & 3 & 6 \\
\hline 3 & 3 & -3 & 6 \\
\hline
\end{tabular}

Figure 5 shows the geographical locations of the cell sites. For testing purposes, a limited number of MTSOs, cell sites and avoiding areas are considered. However, for more realistic problems, the model allows additional quantities of the units. The data used for the coordinates of the locations of MTSOs, cell sites and avoiding areas also can be any positive or negative digits.

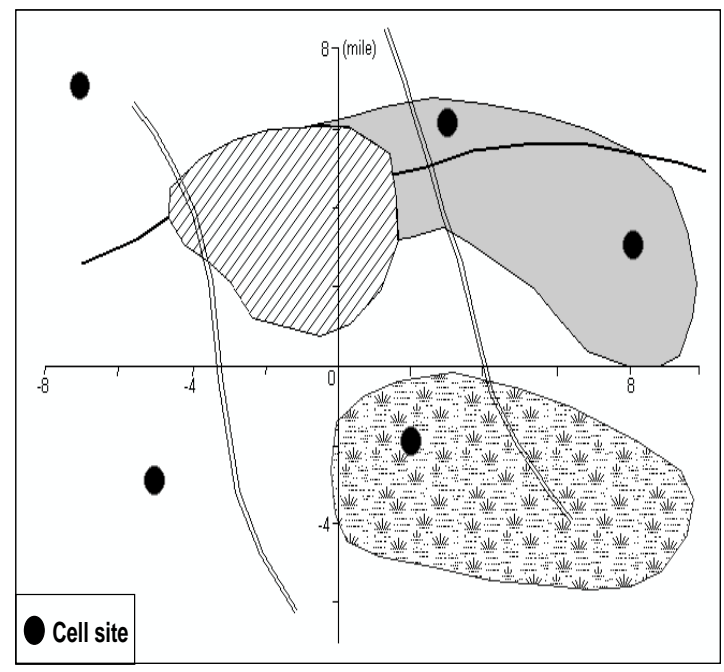

Figure 5: Locations of cell sites

For the constraints of the accessibility issues, an easy-to-access place, such as close to a road, should be selected. However, it is not easy to define easy-to- access places since all points along the road or highways are considered feasible areas as long as they are not in restricted areas. If it is mathematically formulated, it is trivial and time consuming. Since the further the distance from the optimum, the worse the objective value, it is economical to select a potential location where it is closest to the optimal point.

\section{TEST CASES AND THE RESULT ANALYSIS}

\section{Case 1: finding the best fitting location for MTSO without the candidate sites selection technique}

Even though a real restricted area has numerous indentations, such as the shapes of lakes, buildings and residential areas, in general, it is technically challenging and economically inefficient to reflect all the situations mathematically. Hence for economical reasons, most of mathematical models are developed based on the simplified form of a real situation [6]. For simplification purposes, the test case 1 assumes circular avoiding areas. Figure 6 shows the geographical location of optimal MTSO considering the avoiding (restricted) areas.

For the problem with the objective and all the constraints, the Solver ${ }^{\circledR}$ found the coordinates of the optimal location, $(-0.89,-1.88)$ with the objective value of 36.86. The location of MTSO is an edge of a restricted area, and it does not satisfy the accessibility issue. If the accessibility issue is considered, the location is close to a road as the arrow indicates in Figure 6. The place is a least distance from the optimal location. The coordinate for the best fitting location is around a point $(-3,-3)$, which generates the objective value of 41.22 while

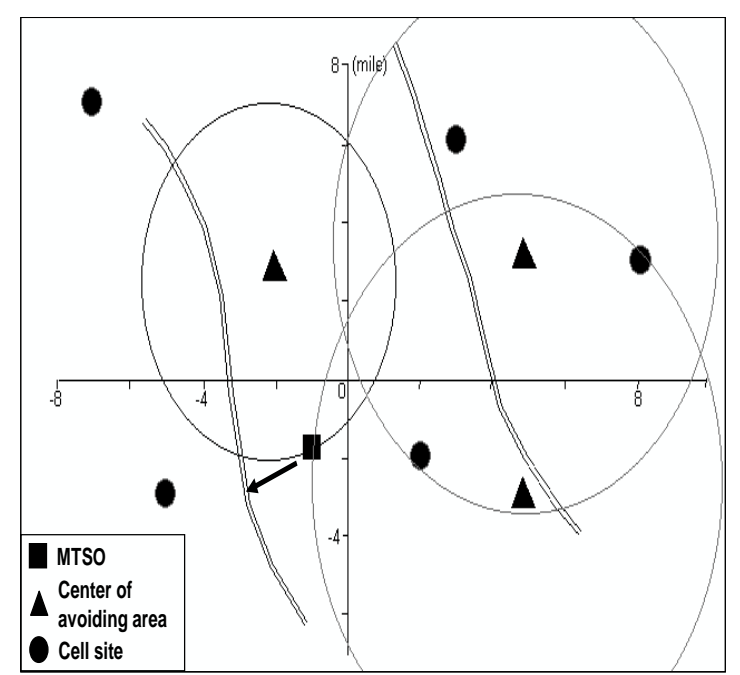

Figure 6: The best fitting location for case 1 


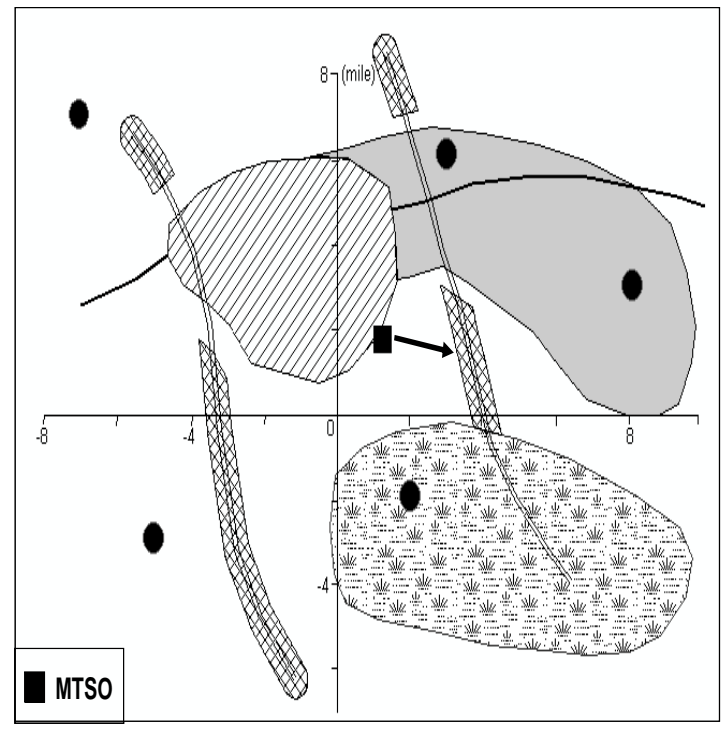

Figure 7: The best fitting location for case 2

satisfying all constraints. Note that the objective value of the best fitting location is greater than that of the optimal location.

\section{Case 2: finding the best fitting location for MTSO with the candidate sites selection technique}

For case 2, first of all, the problem with the objective only is solved using the Solver ${ }^{\circledR}$ and it found the optimal solution. The coordinate of the optimal location is $(1.11,1.89)$ with the objective value of 32.90. Comparing this to the solution of case 1 which is 36.86, the objective value has decreased. In general, if constraints are added in a linear or nonlinear programming model, the objective value is increased in a minimization problem and decreased in a maximization problem [7].

The optimal location for case 2 is not feasible because it violates the constraints of restricted areas and accessibility issues. If the optimal point moves to a feasible point considering the accessibility issues, the besting fitting location is the closest to a road, which is in candidate areas, and the point satisfies all constraints. Figure 7 shows the optimal location, and the best fitting location. The coordinate for the best fitting location is around a point $(4,1.5)$, which generates the objective value of 35.27. Comparing Figure 6 and 7, the best fitting location of case 1 is the area close to the road in the left hand area. However, the best fitting location of case 2 is close to the road in the right hand area. Both locations are feasible, but the final selected locations are different and so are the objective values, 41.22 and 35.27 for case 1 and case 2, respectively. Since the objective is to minimize the distances, case 2 generated the better solution.

\section{SUGGESTED METHOD FOR SELECTING A LOCATION OF MTSO}

As seen in cases 1 and 2, the constraints of restricted areas must be satisfied, but the mathematical model does not require these constraints. The following is the suggested procedure for selecting a MTSO location:

1) Identify the candidate areas and avoiding areas using GIS site selection technique.

2) Find the optimal location by solving the problem without considering the restricted areas. If the solution satisfies all constraints, stop. Otherwise, execute the third step.

3) Find a location which is closer to the optimal solution, considering the accessibility issues.

4) Check the feasibility of the point. If the point is feasible, stop. Otherwise, repeat step 3.

In the second step, the location of the optimal solution is either within or out of a restricted area. However, the best fitting location must not be located in a restricted area, and should have easy accessibility. The suggested method is time efficient because it does not need to define the constraints in the mathematical model. Most of all, the real problem becomes much simpler since the method only considers the areas around the optimal location.

\section{CONCLUSIONS AND FUTURE RESEARCH}

The location of a MTSO in a cellular telecommunication system is affected by factors such as the distances between each component, the area restrictions and the accessibility. The restricted areas and potential areas were identified using GIS site selection technique and a non-linear programming model was developed to find an optimal solution with the above factors. The model was solved and analyzed with two cases: without the candidate sites selection technique and with the candidate sites selection technique.

Base on the test results, case 2 with the candidate sites selection technique generated a better solution. Since the suggested method does not consider the restricted areas in a mathematical model, the method is time efficient and cost effective in terms of computation effort.

An extension of this research would be to further test, verify and validate the suggested model with a real cellular telecommunication network design. Also, the proposed methodology can be extended to solve the 
manufacturing layout design and facility allocation problems, which are known to have common constraints such as cost, distance and capacity.

\section{REFERENCES}

1. Bazaraa, M. S., Sherali, H. D., \& Shetty, C. M. (1993). Nonlinear Programming: Theory and Algorithms (2nd ed.). New York, NY: John Wiley \& Sons, Inc.

2. Chang, K. (2006). Introduction to Geographic Information Systems. $3^{\text {rd }}$ edition, New York, NY: McGraw-Hill.

3. Chang, K., D.L. Verbyla, and J.J. Yeo (1995). Spatial Analysis of Habitat Selection by Sitka Black-Tailed Deer in Southeast Alaska, USA. Environmental Management, 19, 579-89.

4. Dutta, A., \& Kubat, P. (1999). Design of partially survivable networks for cellular telecommunication systems. European Journal of Operational Research, 118, 52-64.

5. Kidner, D., I. Fitzell, P. Rallings, M. A. Nuaimi, and A. Ware (1999). A distributed Approach for Planning Radio Communications. 4th International Conference on GeoComputation. Fredericksburg, VA.

6. Lee, W. C. Y. (1993). Mobile Communications Design Fundamentals. $2^{\text {nd }}$ edition, New York, New York: John Wiley \& sons.

7. Nash, S. G., \& Sofer, A. (1996). Linear and Nonlinear Programming. New York, New York: McGraw-Hill.

8. Palenchar, J. (2005). Cellular subscriber growth breaks 1st-half record. TWICE. Retrieved March 05, 2006, from http://www.twice.com/article/CA6277312.html

9. Rardin, R. L. (1998). Optimization in Operations Research. Upper Saddle River, New Jersey: Prentice Hall.

10. Shay, W. A. (2004). Understanding Communications and Networks (3rd ed.). Belmont, California: Brooks/Cole-Thomson Learning.

11. Stallings, W. (2005). Business Data Communications (5th ed.). Upper Saddle River, New Jersey: Pearson Education.

12. Tierney, J. A. (1975). Calculus and Analytic Geometry (3rd ed.). Boston, Massachusetts: Allyn and Bacon.

13. Tomasi, W. (2005). Introduction To Data Communications and Networking. Upper Saddle River, New Jersey: Pearson Education. 